\title{
Effectiveness of biatrial epicardial application of amiodarone-releasing adhesive hydrogel to prevent postoperative atrial fibrillation
}

\author{
Xiao Dong Feng, MD, ${ }^{a}$ Xue Ning Wang, MD, PhD, ${ }^{\mathrm{b}}$ Xin Hiu Yuan, MD, ${ }^{\mathrm{c}}$ and William Wang, MD, $\mathrm{PhD}^{\mathrm{d}}$
}

Objective: Postoperative atrial fibrillation (POAF) is the most frequent complication arising after cardiac surgery, occurring in $30 \%$ of cases. Amiodarone is the most effective drug for prophylaxis and treatment. However, because of significant extracardiac side effects, only high-risk patients are eligible for prophylactic amiodarone therapy. We performed a randomized prospective study of 100 patients undergoing cardiac surgery with epicardial application of amiodarone-releasing hydrogel to determine the effectiveness of preventing POAF.

Methods: After institutional review board approval, 100 patients, from January 2012 to July 2013, who had undergone cardiac surgery, were randomized to 2 equal groups. The study group received poly-based hydrogel with amiodarone sprayed diffusely over the epicardium. The control group underwent the procedure without the spray. Continuous telemetry monitored for POAF, and amiodarone levels in the atria, plasma, and tissue were measured postoperatively. Daily electrocardiographic parameters were measured until postoperative day 14 .

Results: The incidence of POAF was significantly less in the study group, with 4 of 50 patients $(8 \%)$ incurring atrial fibrillation compared with 13 of 50 patients $(26 \%)$ in the control group $(P<.01)$. The mean amiodarone concentrations in the atria $(12.06 \pm 3.1)$ were significantly greater than those in the extracardiac tissues $(1.32 \pm 0.9 ; P<.01)$. The plasma amiodarone levels remained below the detection limit $(<8 \mu \mathrm{g} / \mathrm{mL})$ during the 14 days of follow-up. Bradycardia was observed less in the study group $(76 \pm 29)$ than in the control group $(93 \pm 18 ; P<.01)$.

Conclusions: Epicardial application of amiodarone-releasing adhesive hydrogel is a less invasive, welltolerated, quick, and effective therapeutic option for preventing POAF at minimal risk of extracardiac adverse side effects. (J Thorac Cardiovasc Surg 2014;148:939-43)

Postoperative atrial fibrillation (POAF) is the most frequent complication arising after cardiac surgery, occurring in $30 \%$ of cases. ${ }^{1,2}$ It increases patient mortality and morbidity, hospitalization duration, and healthcare resource usage. Currently, the available conventional therapies for preventing POAF are suboptimal. Amiodarone, a class III antiarrhythmic medicine, is the most effective drug for prophylaxis and treatment. Clinical studies have demonstrated the efficacy of oral and intravenous amiodarone therapy to prevent POAF. Despite its superior efficacy, amiodarone therapy has several disadvantages, including the requirement of a loading period, interactions with many other drugs, and, most importantly, serious extracardiac side effects, such

\footnotetext{
From Shanghai Eastern Hospital, ${ }^{\mathrm{a}}$ Shanghai, China; ShanXi Cardiovascular Hospital, ${ }^{\mathrm{b}}$ TaiYuan, Shanxi, China; Changzhi Peace Hospital, ${ }^{\mathrm{c}}$ Changzhi, Shanxi, China; and Scripps Memorial Hospital, ${ }^{\mathrm{d}}$ La Jolla, Calif.

Disclosures: Authors have nothing to disclose with regard to commercial support.

Read at the 94th Annual Meeting of The American Association for Thoracic Surgery, Toronto, Ontario, Canada, April 26-30, 2014.

Received for publication March 28, 2014; revisions received May 15, 2014; accepted for publication May 19, 2014; available ahead of print July 18, 2014

Address for reprints: William Wang, MD, PhD, Scripps Memorial Hospital, 9850 Genesee Ave, Suite 560, La Jolla, CA 92037 (E-mail: wangmd100@gmail.com). $0022-5223 / \$ 36.00$

Copyright (c) 2014 by The American Association for Thoracic Surgery http://dx.doi.org/10.1016/j.jtcvs.2014.05.049
}

as thyroid dysfunction, pulmonary toxicity, and hepatic toxicity. ${ }^{3,4}$ These have been, in part, attributed to the relatively high drug concentrations required with systemic administration.

The concept of local amiodarone delivery to the myocardium has been investigated in several animal studies using intrapericardial infusion of amiodarone solutions. ${ }^{5,6}$ They were able to minimize the ventricular and extracardiac adverse effects of amiodarone and made a loading period superfluous. Although the results are encouraging, most arrhythmias require a longer treatment duration. Longterm catheter-based intrapericardial infusion could increase the risk of infection and fibrosis. This approach is also not applicable to the postoperative patient, because the pericardium is usually left open and in communication with a mediastinal drain. Along with bleeding and effusions, any drug solution instilled at surgery would be rapidly evacuated. A few studies researching the effectiveness of the topical application of a biodegradable disc with amiodarone or amiodarone-eluting bilayered patch have reported encouraging results. ${ }^{7,8}$ However the amount of exposure to the atrial epicardial surface is often limited using those applications. Biatrial epicardial application of drug-releasing hydrogel might offer the advantage of a more localized (biatrial-targeted) drug delivery, because, 


\section{Abbreviations and Acronyms \\ $\mathrm{AF}=$ atrial fibrillation \\ $\mathrm{CABG}=$ coronary artery bypass grafting \\ PEG = polyethylene glycol \\ POAF $=$ postoperative atrial fibrillation}

despite diffusion to other heart chambers through leakage to the pericardial fluid, the amiodarone concentration of the biatrial myocardium remains very high.

CoSeal Surgical Sealant (Baxter Healthcare, Deerfield, Ill) is used in a wide range of surgical specialties, including cardiac procedures, primarily for prevention of pericardial adhesions and anastomotic bleeding. These agents consist of 2 biodegradable synthetic polyethylene glycol (PEG) polymers that are mixed at application, forming a strong hydrogel that vigorously adheres to tissue. ${ }^{9}$ These properties make this biomatrix attractive as a vehicle for local drug delivery. Lidocaine mixed with this hydrogel has been shown to reduce postoperative pain after breast augmentation. ${ }^{10}$ The hydrogel has also been used to deliver nerve growth factor, stem cells, and chemotherapeutic agents. ${ }^{11}$ From these studies, we reasoned that the addition of amiodarone to this hydrogel would produce a drug-releasing matrix capable of suppressing atrial tachyarrhythmias and the systemic drug levels would remain low, even in the presence of effusion or pericardial drainage. The purpose of the present prospective study was to evaluate the efficacy of amiodarone-loaded hydrogels applied to the atria to prevent POAF.

\section{METHODS}

A total of 100 patients scheduled to undergo coronary artery bypass grafting (CABG) from January 2012 to July 2013 were randomized and prospectively enrolled in the present study. The institutional review board and ethical research committee approved the study, and all patients provided informed consent. Patients were excluded if they had a history of pacemaker or automatic internal cardiac defibrillator placement or if significant preoperative events developed, such as cardiogenic shock or ventricular tachyarrhythmia. The 100 patients (60 men, 40 women; mean age, $52 \pm 9$ years), who were undergoing conventional on-pump CABG, were randomized to 2 equal groups. The study group received CoSeal-based hydrogel with amiodarone sprayed diffusely over the biatrial epicardium. The control group underwent CABG without amiodarone spraying (Table 1).

A sprayable polymeric matrix, CoSeal Surgical Sealant was obtained from Baxter Healthcare. The kit includes 2 synthetic PEGs: a dilute hydrogen chloride solution and a sodium phosphate/sodium carbonate solution. Amiodarone hydrochloride powder (Sigma-Aldrich, St Louis, Mo), $1 \mathrm{mg} / \mathrm{kg}$ bodyweight, was first added to the PEG powder and then mixed with the solutions until the powder had completely dissolved. These components form a hydrogel. All patients primarily underwent on-pump CABG. Triple radiofrequency pulmonary vein epicardial circumferential isolation was performed in 5 patients who had been diagnosed with paroxysmal atrial fibrillation (AF; 3 in the study group, 2 in the control group). All patients underwent dissection of the superior vena cava-atrial junction area and the free left atrial anterior wall, which was adjacent to the transverse sinus (Backmann's bundle area). Temporary epicardial atrial and ventricular pacing wires were inserted in all patients at the end of surgery. Before the sternum was closed, the hydrogel was sprayed diffusely using a carbon dioxide driver set over the right atrial lateral wall, left atrial appendage, and transverse sinus area. The pericardium was approximated with interrupted sutures, and a single mediastinal chest tube was placed retromediastinally above the pericardium. Myocardial venous blood sampling was obtained from the coronary venous sinus under fluoroscopic control during postoperative day 3. Also, right atrial endomyocardial biopsy was performed from areas adjacent to the fossa ovalis for measurement of the myocardial amiodarone concentration. Finally, abdominal extraperitoneal adipose tissue within the chest tube site was biopsied during chest tube removal on postoperative day 3 . The amiodarone plasma concentrations were also measured using blood drawn from a peripheral vein during postoperative days 2 and 5 . The amiodarone and desethylamiodarone assays were done using the standard method of high-performance liquid chromatography (HP-Series 1090; Hewlett Packard, Palo Alto, Calif). A cardiac enzyme panel was measured on postoperative days 1,3 , and 5 and before discharge to monitor local atrial myocardial injury. Continuous telemetry was used to monitor for POAF. Daily electrocardiographic parameters (RR, PQ, and QT intervals, maximal intervals between the peak and end of the $\mathrm{T}$ wave, and $\mathrm{P}$ and QRS widths) were measured until postoperative day 14. Postoperatively, the patients in the control group received either intravenous or oral amiodarone for new-onset atrial fibrillation. Electrical cardioversion was performed before hospital discharge for any patient who was not in normal sinus rhythm.

All values are presented as the mean \pm standard deviation. Statistical analysis comparing the data between the 2 groups was performed with the chi-square test for categorical variables. Continuous variables were compared using 2-tailed Student $t$ tests and the Kruskal-Wallis test, as appropriate. The collected data were analyzed using the number cruncher statistical systems software (NCSS, Kaysville, Utah).

\section{RESULTS}

No epicardial amiodarone hydrogel-related complications occurred. The greatest incidence of troponin $\mathrm{T}$ detection was observed in 2 patients $(2 \%)$ in the study group with preoperative acute myocardial infarction. Transient bradycardia requiring temporary pacing developed in 7 patients in the study group. The overall hospital mortality was $1 \%$. Stroke occurred in 1 patient $(1 \%)$, and 5 patients underwent reoperation for bleeding $(5 \%)$. The mean follow-up period was $6 \pm 9$ months (range, 1-14). The incidence of POAF was significantly less in the study group, with 4 of 50 patients $(8 \%)$ developing POAF compared with 13 of 50 patients $(26 \%)$ in the control group $(P<.01)$. In the study group, the coronary venous sinus blood concentration of amiodarone $(10.09 \pm 3.6 \mu \mathrm{g} / \mathrm{mL})$ was far greater than that in the peripheral plasma $(1.43 \pm 1.6 \mu \mathrm{g} / \mathrm{mL} ; P<.01)$. The plasma concentrations of both amiodarone and desethylamiodarone remained below detection limits $(<8 \mu \mathrm{g} / \mathrm{mL})$ during the 14 days of follow-up. The right atrial myocardium concentration of desethylamiodarone $(12.06 \pm 3.1 \mu \mathrm{g} / \mathrm{g})$ was significantly greater than that in the abdominal adipose tissue $(1.32 \pm 0.9 \mu \mathrm{g} / \mathrm{g} ; P<.01)$. The mean heart rate averaged $76 \pm 29$ beats/min within 7 days in study group compared with $93 \pm 18$ beats/min in the control group $(P<.01)$. The QT intervals increased from $401 \pm 44 \mathrm{~ms}$ before 
TABLE 1. Patient demographic characteristics

\begin{tabular}{|c|c|c|c|}
\hline Characteristic & Study group & Control group & Total \\
\hline Patients (n) & 50 & 50 & 100 \\
\hline Median age (y) & $52 \pm 9.1$ & $51 \pm 7.9$ & - \\
\hline Sex & & & - \\
\hline Male & 29 & 31 & \\
\hline Female & 21 & 19 & \\
\hline Previous MI & $14(28)$ & $13(26)$ & 27 \\
\hline CHF & $9(18)$ & $12(24)$ & 21 \\
\hline Preoperative AF & $3(6)$ & $2(4)$ & 5 \\
\hline Preoperative IABP & $3(6)$ & $4(8)$ & 7 \\
\hline Previous CABG & $4(8)$ & $6(12)$ & 10 \\
\hline Previous PCI & $27(54)$ & $24(48)$ & 51 \\
\hline Hypertension & $16(32)$ & $11(22)$ & 27 \\
\hline Diabetes & $17(34)$ & $19(38)$ & 36 \\
\hline Chronic lung disease & $22(44)$ & $23(46)$ & 45 \\
\hline PVD & $6(12)$ & $7(14)$ & 13 \\
\hline Previous stroke & $5(10)$ & $8(16)$ & 13 \\
\hline Smoking (previous 6 mo) & $35(70)$ & $39(78)$ & 74 \\
\hline BMI $>30 \mathrm{~kg} / \mathrm{m}^{2}$ & $21(42)$ & $19(38)$ & 40 \\
\hline$\beta$-Blockers & $11(22)$ & $14(28)$ & 25 \\
\hline Calcium channel blockers & $5(10)$ & $7(14)$ & 12 \\
\hline Aspirin & $31(62)$ & $37(74)$ & 65 \\
\hline
\end{tabular}

Data presented as n (\%), unless otherwise noted. Univariate $P=$ NS for all items. $M I$, Myocardial infarction; $C H F$, congestive heart failure; IABP, intra-aortic balloon pump; $C A B G$, coronary artery bypass grafting; $P C I$, percutaneous coronary intervention; $P V D$, peripheral vascular disease; $B M I$, body mass index; $A F$, atrial fibrillation.

amiodarone hydrogel use to $412 \pm 53 \mathrm{~ms}$ after amiodarone therapy, and the rate corrected QT intervals increased from $452 \pm 43 \mathrm{~ms}$ to $463 \pm 37 \mathrm{~ms}(P=.06)$.

\section{DISCUSSION}

The main finding of the present study was that biatrial epicardial application of amiodarone-releasing hydrogel was effective in preventing and lowering the incidence of POAF. The treatment was safe and well tolerated and yielded persistently greater drug concentrations in the atria compared with the extracardiac tissues. The treatment was easy to apply and displayed both biodegradability and immediate tissue adherence, allowing more localized drug delivery.

Atrial fibrillation is a common and undesirable event after cardiac surgery and is an important source of patient morbidity and increased resource usage. The incidence of $\mathrm{AF}$ has been reported at $27.6 \%$ after $\mathrm{CABG}, 48.8 \%$ after mitral valve replacement, $32.9 \%$ after aortic valve replacement, and greater after combined procedures. ${ }^{1}$ Postoperative AF has been associated with an increased hospital stay, early stroke risk, and a reduction in in-hospital and long-term survival. ${ }^{12}$ Although promising nonpharmacologic therapies, in particular, ablation procedures, have been developed, pharmacotherapy remains the cornerstone of AF treatment. At present, the most frequently applied drug worldwide for maintaining sinus rhythm in patients with
$\mathrm{AF}$ is amiodarone, a highly lipophilic, multi-ion channel blocker. ${ }^{13}$ Compared with other contemporary clinically used antiarrhythmic agents, it combines high effectiveness with a low risk of Torsades de pointes. However, the major drawbacks of amiodarone involve its multiple systemic side effects, including thyroid, pulmonary, hepatic, and dermatologic toxicity, and its complex and inconvenient pharmacokinetic profile, including slow and quite extensive tissue accumulation and very long half-life. To improve the effectiveness of the drug and minimize its systemic toxicity, 3 alternative applications for epicardial amiodarone delivery have been developed: intrapericardialinfused amiodarone solutions, epicardially sprayed in situ amiodarone-releasing adhesive hydrogels, and epicardially sutured amiodarone-eluting bilayered patches or discs. ${ }^{14,15}$ A number of studies have investigated local drug delivery of antiarrhythmic agents, including lidocaine, digoxin, amiodarone, $\beta$-blockers, procainamide, ibutilide, sotalol, and flecainide. ${ }^{16,17}$ The pharmacokinetic advantages and significant effects on atrial electrophysiologic parameters were invariably demonstrated. Intrapericardial amiodarone infusion is ideally suited for noncardiac surgery patients with an intact pericardial sac, because it provides a natural drug reservoir and space for epicardial drug delivery. A recent study showed intrapericardial delivery of ibutilide, sotalol, and amiodarone did not result in a greater $\mathrm{AF}$ conversion rate or increased risks of infection, local myocardial fibrosis, or ventricular arrhythmia. ${ }^{18}$ This resulted in part from the amiodarone delivery effects on global ventricular electrophysiology causing QT interval prolongation and a shorter infusion duration. The epicardial amiodarone-eluting bilayered patches or discs allow more localized release of drugs, minimize drug leakage to the pericardial fluid to prevent disruption of ventricular electrophysiology, and produce long-term, sustained drug delivery by the biodegradable matrix. Nevertheless, epicardial patch application was considered somewhat difficult and time-consuming and has limited acceptance at the left atrial wall because of the anatomic complexity of the left atrium. The epicardially sprayed, in situ, PEG-based, amiodarone-releasing adhesive hydrogels used in the present study are biodegradable amiodarone-loaded matrixes that adhere to the epicardium and are able to achieve sustained therapeutic myocardial concentrations. We found the application to be technically simple and quick, and, most importantly, the hydrogel diffusion covered both atrial surfaces without adding any complications. The thin atria seem to be suitable for this delivery method, because of their high surface area/wall thickness ratio.

The present study is the first to clinically demonstrate that biatrial epicardial application of amiodarone-releasing hydrogel significantly reduces the incidence of POAF compared with the control group. The delivery method 
specifically increases the amiodarone concentration of the atrial tissue and thus becomes locally effective for the atrium. The atrial concentration of amiodarone was far greater than that in the other organs, and the blood concentration of amiodarone was below detectable levels. This local high amiodarone concentration was attributed to the intracardial diffusion. Bolderman and colleagues ${ }^{8}$ reported that application of amiodarone-releasing hydrogel to the atrium produced 10 -fold greater epicardial drug concentrations than did endocardial concentrations in an animal study. The present study also found this delivery system minimized drug release into the pericardial fluid, did not appear to disturb the ventricular electrophysiology, and enabled longer lasting site-specific drug delivery. No QT/ rate corrected QT interval prolongation, Torsades de pointes, or significant clinical bradycardia developed.

The PEG-based hydrogel used in the present study displayed both biodegradability and immediate tissue adherence during in situ formation, allowing a more localized drug delivery. Moreover, the clinical safety of epicardial application of this hydrogel has been previously demonstrated. ${ }^{19}$ Amiodarone loading did not affect hydrogel tolerability in the present study. Amiodarone extravasation is not uncommon with peripheral vein infusions, and it can potentially cause serious injury and permanent harm such as tissue necrosis. The toxic effects result from the low $\mathrm{pH}$ of the solution $(\mathrm{pH}, 3.5-4.5)$ and the additives polysorbate and benzyl alcohol. Previous animal studies provided in vivo evidence that topical amiodarone application has no direct toxic affect on the atrial myocardium; however, little is known about the structure and functional impairment of the atrial myocardium after epicardial amiodarone application in humans. In the present study, no significant changes were seen in the positive value of troponin $\mathrm{T}$ release between the study and control groups, and the study group did not appear to have a greater incidence of atrial tachyarrhythmia. This compelling clinical evidence has demonstrated that the epicardial amiodarone application carries an extremely low risk of direct tissue injury. The hydrogel used in the present study also reduced epicardial inflammation, explaining its efficacy in preventing pericardial adhesions after cardiac surgery.

The present study had several limitations. First, we did not evaluate the dose effects of the sustained release of amiodarone from the adhesive hydrogels or the kinetics of amiodarone after its release. Darsinos and colleagues ${ }^{6}$ suggested "intracardiac movement" was a possible mechanism for absorption of pericardial application of amiodarone from that space into the heart tissue. We speculate that a similar mechanism plays an important role in elevating the tissue concentration of amiodarone in the atrium, although the precise mechanism was not investigated in our study. Second, only 1 dose of amiodarone hydrochloride powder and 1 type of hydrogel was used to prepare the drug-loaded hydrogels in the present study. Variations in the hydrogel formulations by changing the polymer chemistry or the altering drug dose or adding epicardial steroid application could result in different drug release characteristics. Third, the present study design was selected in accordance with pilot experiments demonstrating that the surgical sealant did not contribute to the antiarrhythmic or anti-inflammatory effects. No amiodarone was present in the hydrogel applied as a placebo in the control group. Finally, it was more captivating to investigate the continuously monitored biatrial electrophysiologic parameters perioperatively and to measure the atrial effective refractory periods and conduction times.

\section{CONCLUSIONS}

Epicardial application of amiodarone-releasing adhesive hydrogel is a less invasive, well-tolerated, quick, and effective therapeutic option for preventing POAF. These results indicate that this method could be promising in the prevention of POAF with a lower risk of ventricular and systemic side effects compared with intravenous and oral amiodarone administration.

\section{References}

1. Almassi GH, Schowalter T, Nicolosi AC, Aggarwal A, Moritz TE, Henderson WG, et al. Atrial fibrillation after cardiac surgery: a major morbid event? Ann Surg. 1997;226:501-13.

2. Villareal RP, Hariharan R, Liu BC, Kar B, Lee VV, Elayda M, et al. Postoperative atrial fibrillation and mortality after coronary artery bypass surgery. J Am Coll Cardiol. 2004; 43:742-8.

3. Vassallo P, Trohman RG. Prescribing amiodarone: an evidence-based review of clinical indications. JAMA. 2007;298:1312-22.

4. Camm AJ. Safety considerations in the pharmacological management of atrial fibrillation. Int J Cardiol. 2008;127:299-306.

5. Ayers GM, Rho TH, Ben-David J, Besch HR, Zipes DP. Amiodarone instilled into the canine pericardial sac migrates transmurally to produce electrophysiologic effects and suppress atrial fibrillation. J Cardiovasc Electrophysiol. 1996; 7:713-21.

6. Darsinos JT, Karli JN, Samouilidou EC, Krumbholz B, Pistevos AC, Levis GM. Distribution of amiodarone in heart tissues following intrapericardial administration. Int J Clin Pharmacol Ther. 1999;37:301-6.

7. Takeda T, Shimamoto T, Marui A, Saito N, Uehara K, Minakata K, et al. Topical application of a biodegradable disc with amiodarone for atrial fibrillation. Ann Thorac Surg. 2011;91:734-9.

8. Bolderman RW, Hermans JB, Rademakers LM, De Jong MM, Bruin P, Dias AA, et al. Epicardial application of an amiodarone-releasing hydrogel to suppress atrial tachyarrhythmias. Int J Cardiol. 2011;149:341-6.

9. Konertz WF, Kostelka M, Mohr FW, Hetzer R, Hübler M, Ritter J, et al. Reducing the incidence and severity of pericardial adhesions with a sprayable polymeric matrix. Ann Thorac Surg. 2003;76:1270-4.

10. Zhibo X, Miaobo Z. Effect of sustained-release lidocaine on reduction of pain after subpectoral breast augmentation. Aesthet Surg J. 2009;29:32-4.

11. Miura S, Mii Y, Miyauchi Y, Ohgushi H, Morishita T, Hohnoki K, et al. Efficacy of slow-releasing anticancer drug delivery systems on transplantable osteosarcomas in rats. Jpn J Clin Oncol. 1995;25:61-71.

12. Kaireviciute D, Aidietis A, Lip GY. Atrial fibrillation following cardiac surgery: clinical features and preventative strategies. Eur Heart J. 2009;30:410-25.

13. Echahidi N, Pibarot P, O'Hara G, Mathieu P. Mechanisms, prevention, and treatment of atrial fibrillation after cardiac surgery. J Am Coll Cardiol. 2008;51: 793-801.

14. Bolderman RW, Hermans JJ, Rademakers LM, Jansen TS, Verheule S, Van der Veen $\mathrm{FH}$, et al. Intrapericardial delivery of amiodarone and sotalol: atrial 
transmural drug distribution and electrophysiological effects. J Cardiovasc Pharmacol. 2009;54:355-63.

15. Hendrikx M, Mees U, Hill AC, Egbert B, Coker GT, Estridge TD. Evaluation of a novel synthetic sealant for inhibition of cardiac adhesions and clinical experience in cardiac surgery procedures. Heart Surg Forum. 2001;4:204-10.

16. Labhasetwar V, Underwood T, Heil RW Jr, Gallagher M, Langberg J, Levy RJ. Epicardial administration of ibutilide from polyurethane matrices: effects on defibrillation threshold and electrophysiologic parameters. J Cardiovasc Pharmacol. 1994;24:826-40.
17. Kolettis TM, Kazakos N, Katsouras CS, Niokou D, Pappa L, Koulouras V, et al Intrapericardial drug delivery: pharmacologic properties and long-term safety in swine. Int J Cardiol. 2005;99:415-21.

18. Vereckei A, Gorski JC, Ujhelyi M, Mehra R, Zipes DP. Intrapericardial ibutilide administration fails to terminate pacing-induced sustained atrial fibrillation in dogs. Cardiovasc Drugs Ther. 2004;18:269-77.

19. Wallace DG, Cruise GM, Rhee WM, Schroeder JA, Prior JJ, Ju J, et al. A tissue sealant based on reactive multifunctional polyethylene glycol. J Biomed Mater Res. 2001;58:545-55. 